\title{
Evaluación de la calidad de las aulas Moodle en la Universidad de Vic - Universidad Central de Cataluña Quality assessment of Moodle courses at the University of Vic - Central University of Catalonia
}

\author{
Richard Samson ${ }^{1}$, Elena Molina Vicente ${ }^{2}$, Eulàlia Massana Molera ${ }^{3}$ and Anna Ballús Pujol ${ }^{2}$ \\ rsamson@uvic.cat, elena.molina@uvic.cat, eulalia.massana@uvic.cat, anna.ballus@uvic.cat \\ ${ }^{1}$ Facultad de Educación, Traducción y \\ Ciencias Humanas \\ Unidad de Docencia Universitaria y \\ Tecnología Educativa \\ Universidad de Vic - Universidad \\ Central de Cataluña \\ Vic, España \\ ${ }^{2}$ Unidad de Docencia Universitaria y \\ Tecnología Educativa
Universidad de Vic - Universidad Central de Cataluña
Vic, España \\ ${ }^{3}$ Facultad de Empresa y \\ Comunicación \\ Unidad de Docencia Universitaria y \\ Tecnología Educativa \\ Universidad de Vic - Universidad \\ Central de Cataluña \\ Vic, España
}

\begin{abstract}
Resumen- El uso de las TIC en la docencia es buen aliado para una adecuada calidad docente. En el entorno universitario, el profesor goza de cierto grado de libertad para decidir su aplicación. Desde las instituciones se establecen estrategias para potenciar y optimizar este uso. La Unidad de Docencia Universitaria y Tecnología Educativa (UDUTE) y el Vicerrectorado de Ordenación Académica de la Universidad de Vic - Universidad Central de Cataluña (UVic-UCC) han diseñado una herramienta de autoevaluación de la calidad de las aulas virtuales (Autobaremo de las aulas Moodle), que, a la vez de formativa, permite obtener datos para establecer nuevos retos. En esta comunicación se muestran los primeros resultados recogidos de las aulas del curso 2020-2021, se analizan y se obtienen conclusiones que, juntamente con otras acciones, nos permiten mejorar en la docencia.
\end{abstract}

\section{Palabras clave: Moodle, calidad aula virtual, mejora docente}

Abstract- The use of ICT in education is a good ally for quality teaching. In the university sector, the teacher enjoys a certain degree of freedom in deciding how to use resources. Centres establish strategies to promote and optimise ICT use. The University Teaching and Educational Technology Unit (UDUTE) and the Vice-Rectorate for Academic Affairs of the University of Vic - Central University of Catalonia (UVic-UCC) have devised a tool for self-assessment of the quality of Moodle courses, which is a training opportunity and allows data to be obtained in order to prioritise further challenges. In this paper we outline the data collected from courses during the 2020-2021 academic year, we analyse the first results and we arrive at conclusions that, together with other actions, will allow us to continue improving our teaching.

Keywords: Moodle, course quality, teaching improvements

\section{INTRODUCCIÓN}

En la UVic-UCC utilizamos la plataforma de aprendizaje Moodle como herramienta complementaria a las actividades presenciales de las sesiones de clase desde el curso 2012-2013.
En la utilización de herramientas de soporte para mejorar la docencia, Moodle ha sido un gran aliado (aunque no el único) y en la UVic-UCC se han desarrollado diferentes estrategias y acciones para potenciar y optimizar su manejo.

Aunque este uso en ningún caso explica todo lo que pasa en el aula (en sesiones presenciales o virtuales), permite identificar algunos indicadores de cómo se plantea y estructura la docencia en aquella asignatura, por lo que el estudio de cómo la comunidad universitaria maneja Moodle, se considera una herramienta útil para valorar la calidad de la docencia.

En esta comunicación se muestran los resultados de la revisión de la calidad de las aulas en línea a partir de una actividad Questionnaire de Moodle, para el curso 2020-2021. Ésta se plantea como una actividad formativa que permite al profesor reflexionar sobre su práctica docente, ofreciéndole recursos para mejorar el manejo del aula virtual. Además, permite recoger datos que pueden ser usados por parte de la dirección académica y de las diferentes unidades de soporte para el diseño de estrategias que fomenten la mejora de la calidad docente.

Es un proyecto encargado por el Vicerrectorado de Ordenación Académica y puesto en marcha por la UDUTE.

\section{CONTEXTO}

La calidad docente es un aspecto clave para la universidad. La creciente diversificación de estudiantes, y las exigencias de las autoridades educativas y de la sociedad para poder asegurar unas formaciones aplicadas, actualizadas y ágiles, hacen cada vez más necesaria la mejora de la calidad docente. Si en el pasado esta calidad se ha basado exclusivamente en la especialización y pericia del profesorado, hoy en día estos atributos se tienen que complementar con una capacidad metodológica y saber aprovechar las oportunidades de la tecnología educativa. Esta percepción de necesidad de mejora 
competencial docente viene reforzada por las iniciativas nacionales e internacionales relacionadas (p.e. el Marco Europeo para la Competencia Digital de los Educadores, 2017).

El uso de las de las TIC en la docencia son todas aquellas prácticas que utilizan estas herramientas para el proceso de enseñanza-aprendizaje. Diversos autores consideran que son más importantes cómo se aplican estas herramientas que no sus características (Adell y Castañeda, 2010; Coll et al., 2008; Esteve, 2009; CRUE, 2018).

Según diversos autores consultados por Mercader (2018) la competencia digital docente es un aspecto relevante para un óptimo desarrollo de las tareas del profesorado en el aula. El nivel de esta competencia, a pesar de ser tan importante, es muy desigual, dependiendo de diferentes factores.

Así pues, el uso de las TIC en la docencia es considerado por muchos autores como una oportunidad para innovar y mejorar. La transición para la adopción de estas herramientas recibe el nombre de Transformación digital (Priesca, 2016), que se da en todos los ámbitos de la sociedad. En muchos más casos de los deseados, el profesorado muestra resistencia a este cambio (Córica, 2020).

En el entorno universitario, el docente tiene, en general, flexibilidad para decidir qué uso quiere adoptar. Cada institución establece estrategias para intensificar la utilización de las TIC, formación y acompañamiento Esto genera una situación desigual según las universidades, sus estrategias y los recursos de que disponen (CRUE, 2018).

En este contexto la plataforma de aprendizaje Moodle puede jugar el papel de dinamizador, ya que sus actividades se basan en flujos de trabajo que orientan la actividad de los estudiantes, de acuerdo con los preceptos del aprendizaje activo. Por lo tanto, el aprovechamiento adecuado de las actividades de Moodle puede ser un buen indicador del grado de compromiso del profesorado con la formación basada en el protagonismo y participación activa de los estudiantes, conforme a las últimas tendencias pedagógicas y el mismo modelo de formación de la universidad (https://www.uvic.cat/es/universidad/modeloformacion). Las cifras internas de uso en nuestro centro y los resultados de otros investigadores indican que entre las actividades Moodle más significativas para sustentar el trabajo en línea y la interacción son el Foro de mensajes, la Tarea de tramitación de un trabajo individual y el Cuestionario de formularios de preguntas de tipo test (Badia et al. 2019; Hijazi et al. 2020).

\section{DESCRIPCIÓN}

En la UVic-UCC la mayoría de la docencia es presencial, si bien hay algunos grados y másteres semipresenciales y algunos que se desarrollan totalmente en línea. Sea cual sea el formato de docencia, para cada curso académico se da de alta un aula Moodle para cada asignatura de grado o máster, que llamamos aula virtual, aula Moodle o, simplemente, aula.

A partir de las actividades de soporte al profesorado en el uso de Moodle de la UDUTE y en estudios internos anteriores, se ha observado que, si bien cada vez las funcionalidades que permiten estas aulas son más y mejor usadas, aún es muy desigual su aplicación en el conjunto de la universidad.

Así pues, se ha considerado crucial realizar una revisión de la calidad de las aulas Moodle, en función de los indicadores y dimensiones de la encuesta Autobaremo (más abajo), que caracterizan de forma cumulativa el grado de coherencia de la información presentada y el aprovechamiento de las herramientas de la plataforma Moodle. Para ello, la UDUTE y el Vicerrectorado de Ordenación Académica han diseñado la actividad Autobaremo de las aulas Moodle. Se trata de una actividad que recoge el estado de las aulas en línea, a la vez que fomenta la mejora de la prestación docente porque ofrece herramientas, consejos y recursos para que el profesorado aprenda a manejar mejor la plataforma.

Este Autobaremo está configurado como instancia de la actividad Moodle Questionnaire, que es una herramienta de encuesta avanzada

(https://docs.moodle.org/311/en/Questionnaire_module), y consta de 32 indicadores organizados en cuatro dimensiones.

\section{Presentación y orientaciones generales}

2. Espacios de comunicación e información complementaria

3. Documentos y actividades principales

\section{Factores de valor añadido}

En la Tabla 1 se presentan los indicadores que forman parte de cada dimensión. La actividad de Autobaremo incluye una presentación del contexto, unas instrucciones para cumplimentarla y el contacto con la UDUTE por si es necesario resolver dudas. A la vez, se acompañan estos títulos con amplias explicaciones y ejemplos de lo que se le recomienda al profesorado para cada indicador.

El Autobaremo se sugiere al profesorado en formaciones (sobretodo las de personal de nueva incorporación), insistiendo en su parte autoformativa para la mejora de las aulas. A partir del curso 2021-2022 formará parte del proceso de evaluación del profesorado que se presente al programa (voluntario) de obtención de quinquenios de docencia.

Tabla 1

Dimensiones e indicadores de la calidad de las aulas Moodle.

\begin{tabular}{l}
\hline 1. Presentación y orientaciones generales \\
\hline Q02: Estructura visual \\
\hline Q03: Uso de la página principal \\
\hline Q04: Sin espacios vacíos (en la página principal) \\
\hline Q05: Identificación de elementos (títulos específicos) \\
\hline Q06: Numeración de elementos \\
\hline Q07: Ilustración (imagen identificativa de la asignatura) \\
\hline Q08: Plan de trabajo y didáctica \\
\hline Q09: Guía del estudiante \\
\hline 2. Espacios de comunicación e información complementaria \\
\hline \begin{tabular}{l} 
Q10: Tablón del profesorado (avisos) \\
\hline Q11: Tablón en uso (grado de aprovechamiento del Tablón del \\
profesorado)
\end{tabular} \\
\hline Q12: Foro general (para participantes de la asignatura) \\
\hline $\begin{array}{l}\text { Q13: Foro general en uso (Grado de aprovechamiento del Foro } \\
\text { general de la asignatura) }\end{array}$ \\
\hline Q14: Calendario \\
\hline \begin{tabular}{l} 
Q15: Contacto del profesorado \\
\hline
\end{tabular}
\end{tabular}


Q16: Redes sociales

Q17: Otros bloques laterales

\begin{tabular}{l}
\hline 3. Documentos y actividades principales \\
\hline Q18: Formato de documentos (uso de plantillas institucionales) \\
\hline Q19: Atribución (citación de los materiales usados) \\
\hline Q20: Páginas HTML \\
\hline Q21: Multimedia \\
\hline Q22: Calificaciones \\
\hline Q23: Tareas \\
\hline Q24: Retroalimentación en línea (integrada en las Tareas) \\
\hline Q25: Cuestionarios \\
\hline 4. Factores de valor añadido \\
\hline Q26: Enlaces externos/multilingües \\
\hline Q27: Grabación propia \\
\hline Q28: Wikis y colaboración (otras tareas de trabajo en grupo) \\
\hline Q29: Evaluación social (entre iguales) \\
\hline Q30: Compartir trabajos / Glosarios \\
\hline Q31: Recoger datos / Base de datos \\
\hline Q32: Coevaluación / Taller de Moodle \\
\hline Q33: Contenidos interactivos / H5P \\
\hline
\end{tabular}

En los resultados de la actividad, cada indicador tiene un valor de 1, por lo que un aula que los cumpla todos, obtiene una puntuación total de 32 . Es una actividad diseñada para que el profesorado de la UVic-UCC realice su autoanálisis, pero puede incluirse, también, valoraciones externas.

Los datos que aquí se presentan incluyen 37 aulas evaluadas durante el curso 2020-2021 por el propio profesorado. Para obtener una visión más amplia, desde la UDUTE se han evaluado 25 aulas más, con lo que presentamos un total de 62 aulas. Esta muestra contiene como mínimo un aula para cada grado y una de máster para cada facultad.

Con el trabajo que se presenta no se puede valorar la posible diferencia entre el resultado del baremo si lo responde el propio profesor o si lo responde un externo. Aun así, usaremos conjuntamente las respuestas de unos y otros.

La muestra utilizada no permite obtener resultados significativos, ya que la UVic-UCC dispone de más de 2.500 aulas. Pero sí que nos sirve como prueba piloto para su implantación más intensiva y para observar tendencias de este profesorado.

\section{Resultados}

A partir de los datos obtenidos se analizan cualitativamente y se representan descriptivamente las medianas obtenidas para cada indicador de cada dimensión, de manera global para las 62 aulas revisadas en la UVic-UCC para el curso 2020-2021.

\section{A. Presentación y orientaciones generales}

En la Figura 1 se muestran gráficamente las medianas obtenidas para los indicadores de la dimensión Presentación y orientaciones generales.

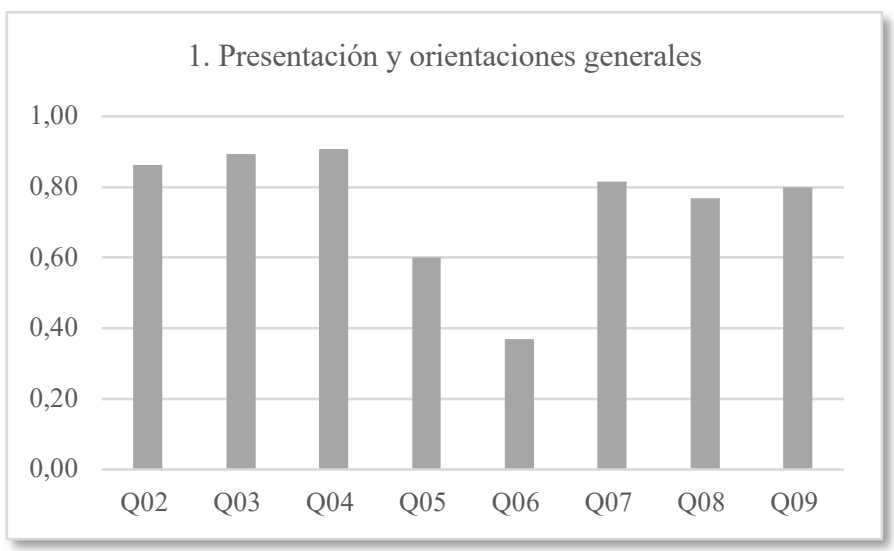

Figura 1. Medianas de los indicadores de la dimensión Presentación y orientaciones generales (curso 2020-2021, universidad UVic-UCC).

Se observa que, en general, el profesorado tiene en cuenta la disposición del aula, sin dejar espacios vacíos, que incluye una imagen representativa de la asignatura y que incorpora los documentos básicos como son la Guía del estudiante y el Plan de trabajo.

En cambio, vemos que hay que incidir más en que los títulos de las secciones, recursos y actividades estén bien definidos y contextualizados (evitando nombres genéricos o poco informativos).

\section{B. Espacios de comunicación e información complementaria}

En la Figura 2 se muestran gráficamente las medianas obtenidas para los indicadores de la dimensión Espacios de comunicación e información complementaria.

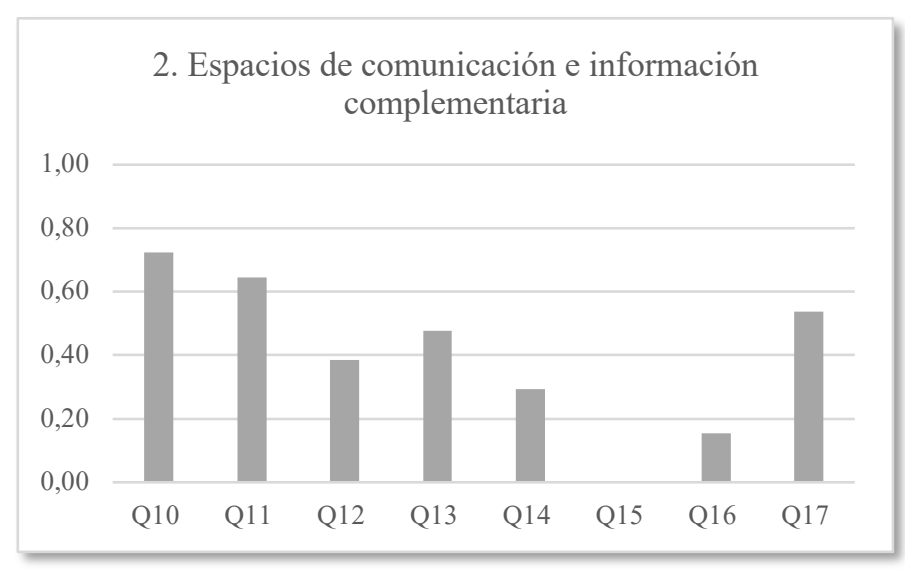

Figura 2. Medianas de los indicadores de la dimensión Espacios de comunicación e información complementaria (curso 2020-2021, universidad UVic-UCC).

Se observa que en general existe un tablón de avisos y noticias y un foro general, pero que se usan poco para la comunicación a través del aula. Por lo tanto, podemos decir que mayoritariamente las aulas virtuales son más un repositorio de documentos y actividades que no un espacio de interacción. 
A menudo no hay la identificación del profesorado al cargo de la asignatura y no siempre es fácil encontrar la información básica de contacto con éste.

\section{Documentos y actividades principales}

En la Figura 3 se muestran gráficamente las medianas obtenidas para los indicadores de la dimensión Documentos y actividades principales.

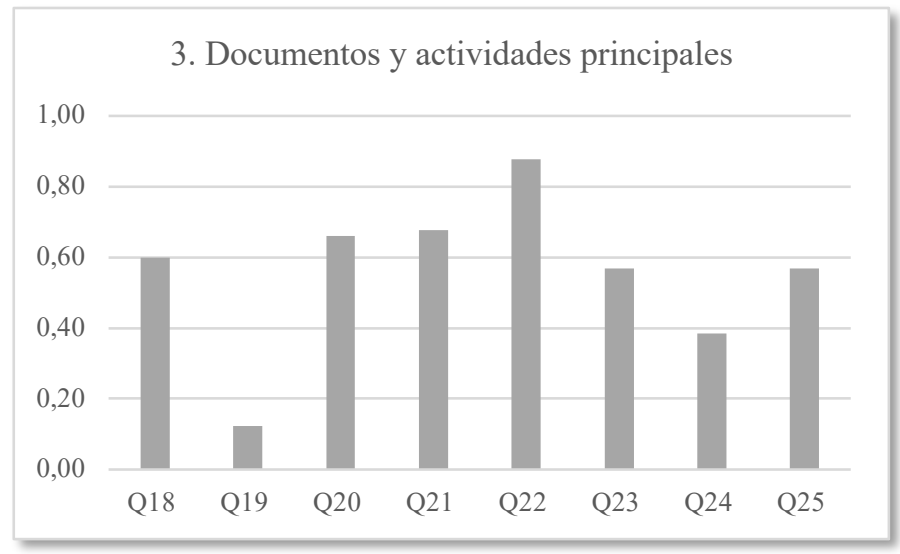

Figura 3. Medianas de los indicadores de la dimensión Documentos y actividades principales (curso 2020-2021, universidad UVic-UCC).

Se observa que las aulas se nutren sobre todo de documentos en pdf elaborados por el profesorado para los que la UVic-UCC dispone de unas plantillas que no son siempre utilizadas. En la mayoría de los casos no se hace una atribución correcta de las imágenes o fuentes de origen de elementos multimedia.

También se usan apuntes en formato Página (html), que favorecen la edición y actualización de contenidos formativos.

Con respecto a las actividades, se usa sobre todo la Tarea de Moodle, pero en muchos casos no se aprovecha su sistema de retroacción.

Las calificaciones están bien configuradas en general y se usan Cuestionarios de Moodle tanto para evaluaciones formativas como sumativas.

\section{Factores de valor añadido}

En la Figura 4 se muestran gráficamente las medianas obtenidas para los indicadores de la dimensión Factores de valor añadido.

Esta parte del baremo recoge una variabilidad de uso de recursos que consideramos complementarios. No es de extrañar, pues, que en docencia básicamente presencial sean los que obtienen menor puntuación (con diferencia) en este baremo.

Aún teniendo en cuenta esto, sí se observa el uso de enlaces a fuentes externas $y$ hay un aumento significativo de grabaciones hechas por el propio profesorado y también del uso de herramientas de evaluación social y coevaluación, respecto a otras observaciones anteriores.

\section{Factores de valor añadido}

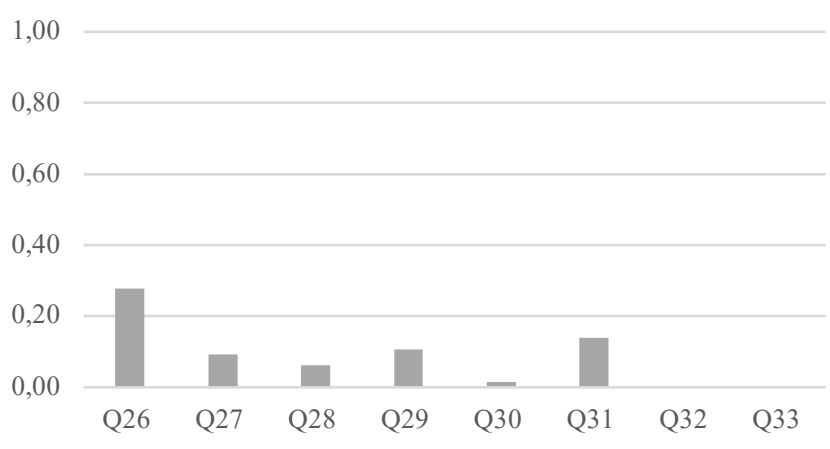

Figura 4. Medianas de los indicadores de la dimensión Factores de valor añadido (curso 2020-2021, universidad UVic-UCC).

\section{CONCLUSIONES}

Este trabajo es solo el primer paso para un análisis más completo y global. Aun teniendo en cuenta la doble fuente de los datos (del propio profesorado y de técnicos) y la posible afectación de esto en los resultados, podemos observar tendencias sobre la calidad de las aulas Moodle en la universidad UVic-UCC para el curso 2020-2021.

\section{A. Fortalezas}

- El aula virtual ha tomado protagonismo y ha sido una herramienta importante del profesorado para sustentar la docencia en línea,

- Sobre todo, se usa para distribuir documentación de interés académico para el estudiante.

- La estructura de las aulas acostumbra a ser correcta y está identificada con imágenes representativas.

- $\quad$ El uso del calificador, en general, es correcto (aunque en algunos casos hay que optimizarlo).

- Se percibe un incremento de uso en las tareas para entregar documentación y de los cuestionarios (evaluativos y sumativos).

- Aumenta el uso de actividades de valor añadido en el aula que permiten una gestión del trabajo en grupo, la autoevaluación y otras actividades sociales.

\section{B. Debilidades}

- Infrautilización de los espacios de comunicación del aula: tablón del profesorado, foro general y retroacción de tareas.

- Poca utilización de las plantillas institucionales de documentación (documentos de texto, presentaciones, piezas audiovisuales).

- Falta de referenciación correcta de la autoría en materiales de terceros (imágenes, sobre todo).

- Poca uniformidad en el nombre de los documentos y secciones. 
- Falta de experiencia en uso de recursos digitales por parte del profesorado.

- Desconocimiento de las oportunidades que ofrece Moodle por parte del profesorado.

- Contexto incierto: adaptación de normativas, nuevos espacios docentes, cambios de modalidades de impartición (presencialidad / semipresencial / modelo híbrido / en línea), entre otros.

\section{Oportunidades}

- Buena predisposición e implicación al aprendizaje por parte del profesorado.

- Oferta de formación interna amplia y adaptable.

- Posibilidad de atención personalizada por parte de la UDUTE.

- Posibilidad de usar Learning Analytics para generar informes regulares automáticos basados en muchos de estos criterios y así identificar a tiempo situaciones de riesgo. Es decir, que se podría confeccionar, para las coordinaciones de las titulaciones y las jefaturas de estudios de los centros docentes, cuadros de mando para controlar la mejora de la docencia de forma permanente.

Un aula virtual bien organizada, con información estructurada, con recursos externos que ayuden a ampliar conocimientos, con actividades relacionadas, de interés para los alumnos, $\mathrm{y}$ con comunicación $\mathrm{y}$ retroalimentación, es fundamental para favorecer una enseñanza de calidad.

Si potenciamos el uso del autobaremo podemos ayudar al profesorado a tomar consciencia de qué aspectos son importantes y hay que tener en cuenta para optimizar el uso del aula virtual y así mejorar su docencia, sea presencial o en línea.

Este trabajo es un primer paso para sistematizar este proceso de mejora continua de la calidad docente. Evidentemente, se tendrá que ir acompañado de otras medidas de reconocimiento de la carrera docente, del desarrollo profesional, de la innovación docente, de provisionamiento de recursos humanos y de la consolidación de buenas prácticas.

\section{REFERENCIAS}

Badia, A., Martín, D., \& Gómez, M. (2019). Teachers' perceptions of the use of Moodle activities and their learning impact in secondary education. Technology, Knowledge and Learning, 24(3), 483-499.

Coll, C., Mauri, T. y Onrubia, J. (2008). Análisis de los usos reales de las TIC en contextos educativos formales: una aproximación sociocultural en Revista Electrónica de Investigación Educativa, 10 (1). Recuperado en http://redie.uabc.mx/vol10no1/contenido-coll2.html

Córica, J. L. (2020). Resistencia docente al cambio: Caracterización y estrategias para un problema no resuelto. RIED. Revista Iberoamericana de Educación a Distancia, 23(2), 255-272.

CRUE (2018). Informe de la situación de las tecnologías educativas en las universidades españolas (FOLTE). Recuperado el 24/05/2021 en https://tic.crue.org/wpcontent/uploads/2020/09/Tecnologias-Educativas2018_DIGITAL.pdf

Esteve, F. (2009). Bolonia y las TIC: de la docencia 1.0 al aprendizaje 2.0, en La Cuestión Universitaria, 5, 58-67.

Hijazi, H., Al-Kateb, G., \& Alkhawaldeh, E. (2020). Investigating the Awareness and Usage of Moodle Features at Hashemite University. IJACSA) International Journal of Advanced Computer Science and Applications, 11(6).

Mercader Juan, C. (2018). Las tecnologías digitales en la docencia universitaria. Barreras para su integración. Tesis doctoral. http://hdl.handle.net/10803/662771

Redecker, C. (2017). Marco europeo para la competencia digital de los educadores. DigCompEdu. Secretaría General Técnica. Centro de Publicaciones. Ministerio de Educación y Formación Profesional.

Priesca, P. (2016). La transformación digital de las empresas. Recuperado el 10 de octubre de 2018 en https://www.fundacionctic.org/ctic/articulos-y-otraspublicaciones/la-transformacion-digital-de-las-empresas 